\title{
Preface to the special issue on personality in personalized systems
}

\author{
Marko Tkalčič ${ }^{1,2}$ - Daniele Quercia ${ }^{3}$. \\ Sabine Graf ${ }^{4}$
}

(C) Springer Science+Business Media Dordrecht 2016

\section{Introduction}

In order to adapt to the current user, adaptive interactive computer systems must acquire the user characteristics that account for the differences between users. In the last two decades, research has been done in mining characteristic features from user traces on the web, sensors and other modalities, which were in turn integrated into user models used in personalized systems.

The psychological concept of personality accounts for individual differences in the users' enduring emotional, interpersonal, experiential, attitudinal, and motivational styles (McCrae and John 1992). The five-factor model (FFM), which is composed of the factors Openness, Conscientiousness, Extraversion, Agreeableness and Neuroticism, is one of the most known models of personality. Having its roots in the lexical theory, it accounts for individual differences in a variety of fields. Research has shown

\arko Tkalčič

marko.tkalcic@gmail.com

Daniele Quercia

daniele.quercia@gmail.com

Sabine Graf

sabineg@athabascau.ca

1 Department for Computational Perception, Johannes Kepler University, Altenberger Strasse 69, Linz, Austria

2 Present Address: Free University of Bolzano Faculty of Computer Science, Piazza Domenicani 3, Bolzano, Italy

3 Bell Labs Cambridge, Cambridge, UK

4 School of Computing and Information Systems, Athabasca University, 1100, 10011-109 Street, Edmonton, AB T5J3S8, Canada 
that personality factors have significant correlations with media content, such as music (Rentfrow and Gosling 2003; Chamorro-Premuzic and Furnham 2007), which made it a good candidate for personalization techniques. Early research in the usage of personality in personalization has been done in addressing the new user problem in recommender systems (Tkalčič et al. 2009; $\mathrm{Hu}$ and $\mathrm{Pu} 2010$ ).

However, until a few years ago, the acquisition of personality was possible only through extensive questionnaires, such as the big five inventory (John and Srivastava 1999). It was the success of methods for the unobtrusive acquisition of personality, mostly form social media (Golbeck et al. 2011; Quercia et al. 2011), that allowed for a quicker and more large-scale acquisition of personality.

Since then, methods for the acquisition of personality and its usage in interactive systems have reached a level of maturity that is manifested through (i) an increasing number of papers published at major venues (such as Kosinski et al. 2013), (ii) a number of dedicated venues, such as the Emotions and Personality in Personalized Systems (EMPIRE) workshop series in 2013, 2014 and 2015, or the Workshop on Computational Personality Recognition (WCPR) in 2013 and 2014, (iii) availability of large-scale datasets, such as the myPersonality (Kosinski et al. 2015), (iv) live personality detection tools, such as the IBM Personality Insights ${ }^{1}$ (Badenes et al. 2014) and (v) a manifesto on personality computing as a research domain (Vinciarelli and Mohammadi 2014).

This special issue presents a snapshot of the state-of-the-art in the unobtrusive acquisition of personality and its usage across a diverse range of personalization applications.

\section{Papers in the special issue}

This special issue contains articles that describe the state of the art in (i) automatic and unobtrusive personality acquisition from social media (Farnadi et al. 2016) and mobile phone data (Lepri et al. 2016) (ii) personality-based user models in behavioral change applications (Lepri et al. 2016), intelligent tutoring systems (Harley et al. 2016) and games (Cowley and Charles 2016), and (iii) usage of personality in recommender systems (Fernández-Tobías et al. 2016).

The paper Computational Personality Recognition in Social Media by Farnadi et al. (2016) deals with the automatic inference of personality traits from users' social media traces. As such, it lies at the base of the chain of personality usage in personalized systems. The authors perform a comparative analysis of computational recognition methods based on textual social media traces from Facebook, Twitter and YouTube (such as status updates, tweets and transcripts of video blogs). The authors identified decision trees and the Linguistic Inquiry and Word Count (LIWC) as the best combination of regressor and feature set. However, they point out that the outcomes may depend on the underlying data.

The paper The Role of Personality in Shaping Social Networks and Mediating Behavioral Change by Lepri et al. (2016) addresses two related issues: (i) personal-

\footnotetext{
1 https://watson-pi-demo.mybluemix.net/
} 
ity inference from mobile phone data and (ii) the role of personality as a mediator between social strategies and behavioral changes (increasing physical activity). Based on a set of 8 -weeks data of 53 subjects the authors developed a methodology for personality inference from ego-network (network consisting of a central node and its direct connections) features. Furthermore, the authors showed that Neuroticism and Extraversion have a mediating effect on behavioral changes. In fact, people who scored high on Extraversion and those who scoreed high on Neuroticism showed an increased behavioral change when subjected to social comparison and peer pressure.

The paper Examining the predictive relationship between personality and emotion traits and students' agent-directed emotions: towards emotionally-adaptive agentbased learning environments by Harley et al. (2016) investigates how personality traits and trait emotions (i.e. trait anger and trait anxiety) are related to agent-directed emotions and learning in an agent-based learning environment. Based on a user study of 123 students, the authors conclude that Agreeableness, Conscientiousness, Neuroticism as well as trait Anger and trait Anxiety were significant predictors of four agent-directed emotions (Enjoyment, Pride, Boredom and Neutral), though relationships differed between pedagogical agents.

The paper Alleviating the New User Problem in Collaborative Filtering by Exploiting Personality Information by Fernández-Tobías et al. (2016) is concerned with the usage of personality in recommender systems, more specifically, to address the new user problem. This is a known issue in recommender systems. When a new user joins the system, there are not enough ratings to make good recommendations. The authors tackle this problem by using personality in three ways: (i) by including it in the matrix factorization approach, (ii) by incorporating it into the active learning approach and (iii) by using it in the cross-domain approach. Based on extensive offline experiments the authors conclude that when a complementary domain is available the cross-domain approach yields better recommendations. When only one domain is available, active learning should be used.

The paper Behavlets: A Method for Practical Player Modelling Using Psychologybased Player Traits and Domain Specific Features by Cowley and Charles (2016) deals with personality in computer games players modeling. The authors introduce the term behavlets for describing a set of features that describe the behavior of game players based on the temperament theory of personality. These features, along with game design patterns, provide the basis for the modeling of users and their playing patterns. The authors show the usage of behavlets on a set of 100 players in the well-known Pac-Man game.

\section{Outlook}

In order to bring the usage of personality to real-world applications, two requirements have to be met: (i) accurate unobtrusive acquisition and (ii) robust user models.

The papers in this special issue (Farnadi et al. 2016; Lepri et al. 2016), as well as in related literature (Kosinski et al. 2013), show that digital traces of user behavior are a good source for automatic personality detection. There is still room for improvement as there are other modalities that have not shown their full potential yet, such as 
image-based personality recognition (see (Segalin et al. 2016; Celli et al. 2014) as early work on the subject), voice-based personality recognition (Mohammadi and Vinciarelli 2015) and fusing various modalities (Skowron et al. 2016).

As the amount of available data grows, so do user models and adaptation techniques. The papers in this special issue (Fernández-Tobías et al. 2016; Cowley and Charles 2016; Harley et al. 2016; Lepri et al. 2016) illustrate some of the approaches that can be taken to design personality-based user models and adaptive systems.

\section{References}

Badenes, H., Bengualid, M.N., Chen, J., Gou, L., Haber, E., Mahmud, J., Nichols, J.W., Pal, A., Schoudt, J., Smith, B.A., Xuan, Y., Yang, H., Zhou M.X.: System U : automatically deriving personality traits from social media for people recommendation. Proceedings of the 8th ACM Conference on Recommender systems, pp. 373-374 (2014)

Celli, F., Bruni, E., Lepri B.: Automatic personality and interaction style recognition from facebook profile pictures. In: Proceedings of the ACM International Conference on Multimedia-MM '14, pp. 11011104 (2014)

Chamorro-Premuzic, T., Furnham, A.: Personality and music: can traits explain how people use music in everyday life? Br. J. Psychol. (London, England : 1953), 98:175-185 (2007)

Cowley, B., Charles, D.: Behavlets: a method for practical player modelling using psychology-based player traits and domain specific features. User Model. User-Adapt. Interact. 4018, 1-68 (2016)

Farnadi, G., Sitaraman, G., Sushmita, S., Celli, F., Kosinski, M., Stillwell, D., Davalos, S., Moens, M-F., De Cock M.: Computational personality recognition in social media. User Model. User-Adapt. Interact. (Special Issue on Personality in Personalized Systems), (2016)

Fernández-Tobías, I., Braunhofer, M., Elahi, M., Ricci, F., Cantador, I.: Alleviating the new user problem in collaborative filtering by exploiting personality information. User Model. User-Adapt. Interact. (2016)

Golbeck, J., Robles, C., Turner, K.: Predicting personality with social media. Proceedings of the 2011 Annual Conference Extended Abstracts on Human Factors in Computing Systems-CHI EA '11, p. 253 (2011)

Harley, J.M., Carter, C.K., Papaionnou, N., Bouchet, F., Landis, R.S., Azevedo, R., Karabachian, L.: Examining the predictive relationship between personality and emotion traits and students' agent-directed emotions: towards emotionally-adaptive agent-based learning environments. User Model. User-Adapt. Interact. (2016)

$\mathrm{Hu}, \mathrm{R} ., \mathrm{Pu}, \mathrm{P} .:$ Using personality information in collaborative filtering for new users. In: Proceedings of the 2nd ACM RecSys'10 Workshop on Recommender Systems and the Social Web, pp. 17-24 (2010)

John, O., Srivastava, S.: The big five trait taxonomy: history, measurement, and theoretical perspectives. In: Pervin, L.A., John, O.P. (eds.) Handbook of Personality: Theory and Research, 2nd edn, pp. 102-138. Guilford Press, New York (1999)

Kosinski, M., Matz, S.C., Gosling, S.D.: Facebook as a research tool for the social sciences. Am. Psychol. 70(6), 543-556 (2015)

Kosinski, M., Stillwell, D., Graepel, T.: Private traits and attributes are predictable from digital records of human behavior. Proc. Natl. Acad. Sci. U.S.A. 110(15), 5802-5805 (2013)

Lepri, B., Staiano, J., Shmueli, E., Pianesi, F., Pentland, A.: The role of personality in shaping social networks and mediating behavioral change. User Model. User-Adapt. Interact. Accepted (Special Issue on Personality in Personalized Systems) (2016)

McCrae, R.R., John, O.P.: An introduction to the five-factor model and its applications. J. Personal. 60(2), p175-215 (1992)

Mohammadi, G., Vinciarelli, A.: Automatic attribution of personality traits based on prosodic features. ACII 2015 Affective Computing and Intelligent Interaction, pp. 29-32 (2015)

Quercia, D., Kosinski, M., Stillwell, D., Crowcroft, J.: Our twitter profiles, our selves: predicting personality with twitter. In: Proceedings-2011 IEEE International Conference on Privacy, Security, Risk and Trust and IEEE International Conference on Social Computing, PASSAT/SocialCom 2011, pp. 180-185. IEEE. (2011) 
Rentfrow, P.J., Gosling, S.D.: The do re mi's of everyday life: the structure and personality correlates of music preferences. J. Personal. Soc. Psychol. 84(6), 1236-1256 (2003)

Segalin, C., Perina, A., Cristani, M., Vinciarelli, A.: The pictures we like are our image: continuous mapping of favorite pictures into self-assessed and attributed personality traits. IEEE Trans. Affect. Comput. 3045, 1 (2016)

Skowron, M., Ferwerda, B., Tkalčič, M., Schedl, M.: Fusing social media cues : personality prediction from twitter and instagram. In: WWW'16 Companion (2016)

Tkalčič, M., Kunaver, M., Tasič, J., Košir, A.: Personality based user similarity measure for a collaborative recommender system. In: Christian, P., Elizabeth, C., Lesley, A., Harry, A., Agius, S., Balaam, M. (eds.) 5th Workshop on Emotion in Human-Computer Interaction-Real World Challenges. Fraunhofer Verlag, Stuttgart (2009). http://publica.fraunhofer.de/documents/N-113443.html

Vinciarelli, A., Mohammadi, G.: A survey of personality computing. IEEE Trans. Affect. Comput. 3045, 1 (2014) 